\title{
Acknowledgements
}

The Journal offers its special thanks to our peer reviewers -

Paul Bramadat

John Costello

James Cresswell

Joseph deFeo

Mario D'Souza
Joana Fraser

Marion Grau

Paul Heidebrecht

Matthew Hoven

Chris Hrynkow
Teresita Kambeitz

Michalene King

Darrell McLaughlin

John Thompson

\section{Issue Statistics}

\section{A. Authors and Submissions}

\begin{tabular}{|l|r|}
\hline Authors and Co-Authors & 18 \\
\hline University-based & 2 \\
\hline Community partners & 20 \\
\hline Total & \\
\hline
\end{tabular}

\begin{tabular}{|l|r|}
\hline Article Submissions & 23 \\
\hline Original proposals for peer and editor review & 3 \\
\hline Articles submitted for editor review & 7 \\
\hline Articles submitted for peer review & 4 \\
\hline Peer-reviewed articles accepted for publication & 3 \\
\hline Editor-reviewed articles accepted for publication & 6 \\
\hline Book reviews submitted for editor review & 5 \\
\hline Book reviews accepted for publication & \\
\hline
\end{tabular}

\begin{tabular}{|l|r|}
\hline Geographic Distribution (Corresponding Authors Only) & \\
\hline Eastern Canada & 1 \\
\hline Non-University Based & 3 \\
\hline Western Canada & 3 \\
\hline St. Thomas More College & 1 \\
\hline University of Saskatchewan & 1 \\
\hline International & 1 \\
\hline Universitas Islam Negeri Sunan Ampel, Indonesia & $\mathbf{1 0}$ \\
\hline University of Liverpool & \\
\hline Non-University Based & \\
\hline Total & \\
\hline
\end{tabular}




\section{B. Peer-Reviewers and Peer-Reviewing}

\begin{tabular}{|l|r|}
\hline Peer Reviewers & \\
\hline Total invitations to peer review & 32 \\
\hline Number of peer reviewers who accepted invitations & 14 \\
\hline
\end{tabular}

\begin{tabular}{|l|r|}
\hline Geographic Distribution (Peer Reviewers) & \\
\hline Eastern Canada & 1 \\
\hline Regis College & 1 \\
\hline St. Michael's College & 1 \\
\hline Conrad Grebel University College & 1 \\
\hline Western Canada & 1 \\
\hline Booth University College & 3 \\
\hline Simon Fraser University & 1 \\
\hline St. Thomas More College & 1 \\
\hline University of Alberta & 1 \\
\hline University of Victoria & 1 \\
\hline Non-University Based & 1 \\
\hline International & 1 \\
\hline Norwegian School of Theology, Oslo & $\mathbf{1 4}$ \\
\hline Duquesne University, US & \\
\hline Fairfield University, US & \\
\hline Total & \\
\hline
\end{tabular}

